Supplement of Atmos. Chem. Phys., 14, 10283-10298, 2014

http://www.atmos-chem-phys.net/14/10283/2014/

doi:10.5194/acp-14-10283-2014-supplement

(C) Author(s) 2014. CC Attribution 3.0 License.

(c) (i)

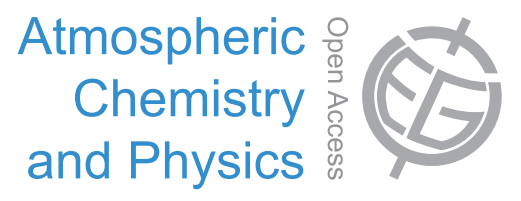

Supplement of

\title{
Linking climate and air quality over Europe: effects of meteorology on $\mathbf{P M}_{2.5}$ concentrations
}

\section{A. G. Megaritis et al.}

Correspondence to: S. N. Pandis (spyros@chemeng.upatras.gr) 
Table S1. Summary of expected effects on total $\mathrm{PM}_{2.5}$ concentrations due to changes on meteorological parameters during the modelled summer, the modelled winter and the modelled fall period.

\begin{tabular}{|c|c|c|c|c|}
\hline $\begin{array}{l}\text { Meteorological } \\
\text { Parameter }\end{array}$ & $\begin{array}{l}\text { Expected } \\
\text { Change of } \\
\text { Parameter }\end{array}$ & $\begin{array}{l}\text { Process } \\
\text { Affected }\end{array}$ & $\begin{array}{l}\text { Predicted Sensitivity } \\
\text { Mean }(10 \%, 90 \%)\end{array}$ & $\begin{array}{l}\text { Expected Concentration } \\
\text { Change }\left(\mathrm{ng} \mathrm{m}^{-3}\right) \\
\text { Mean }(10 \%, 90 \%)\end{array}$ \\
\hline \multicolumn{5}{|c|}{ Summer Period } \\
\hline \multirow{4}{*}{ Temperature } & \multirow{4}{*}{$+1-5.5 \mathrm{~K}^{\mathrm{a}}$} & $\begin{array}{l}\text { BVOC } \\
\text { emissions }\end{array}$ & $\begin{array}{l}10.2 \mathrm{ng} \mathrm{m}^{-3} \mathrm{~K}^{-1} \\
(-13,44)\end{array}$ & $\begin{array}{l}10.2 \text { to } 56.1 \\
(-71.5 \text { to } 242)\end{array}$ \\
\hline & & $\begin{array}{l}\text { Aerosol } \\
\text { partitioning }\end{array}$ & $\begin{array}{l}-49 \mathrm{ng} \mathrm{m}^{-3} \mathrm{~K}^{-1} \\
(-142,4.1)\end{array}$ & $\begin{array}{l}-49 \text { to }-270 \\
(-781 \text { to } 23)\end{array}$ \\
\hline & & Reaction rates & $\begin{array}{l}26.3 \mathrm{ng} \mathrm{m}^{-3} \mathrm{~K}^{-1} \\
(-1.1,49)\end{array}$ & $\begin{array}{l}26 \text { to } 145 \\
(-6.1 \text { to } 270)\end{array}$ \\
\hline & & Overall & $\begin{array}{l}-23.3 \mathrm{ng} \mathrm{m}^{-3} \mathrm{~K}^{-1} \\
(-145,67)\end{array}$ & $\begin{array}{l}-23 \text { to }-128 \\
(-797 \text { to } 369)\end{array}$ \\
\hline \multirow[t]{2}{*}{ Wind Speed } & \multirow[t]{2}{*}{-10 to $10 \%{ }^{b}$} & $\begin{array}{l}\text { Overall } \\
\text { (constant } \\
\text { emissions) }\end{array}$ & $\begin{array}{l}-41.3 \mathrm{ng} \mathrm{m}^{-3} \%^{-1} \\
(-109,0.7)\end{array}$ & $\begin{array}{l}-413 \text { to } 413 \\
(-1090 \text { to } 1090)\end{array}$ \\
\hline & & $\begin{array}{l}\text { Sea salt } \\
\text { emissions }\end{array}$ & $\begin{array}{l}39.2 \mathrm{ng} \mathrm{m}^{-3} \\
(-1.3,107)\end{array}$ & $\begin{array}{l}-392 \text { to } 392 \\
(-1070 \text { to } 1070)\end{array}$ \\
\hline $\begin{array}{l}\text { Absolute } \\
\text { humidity }\end{array}$ & +5 to $40 \%^{\mathrm{c}}$ & Overall & $\begin{array}{l}8.2 \mathrm{ng} \mathrm{m}^{-3} \%^{-1} \\
(-13,27)\end{array}$ & $\begin{array}{l}41 \text { to } 330 \\
(-520 \text { to } 1080)\end{array}$ \\
\hline $\begin{array}{l}\text { Precipitation } \\
\text { rate }\end{array}$ & -40 to $+40 \%^{\mathrm{b}}$ & Overall & $\begin{array}{l}-12.9 \mathrm{ng} \mathrm{m}^{-3} \%^{-1} \\
(-40,-1.6)\end{array}$ & $\begin{array}{l}-516 \text { to } 516 \\
(-1600 \text { to } 1600)\end{array}$ \\
\hline $\begin{array}{l}\text { Precipitation } \\
\text { area }\end{array}$ & -40 to $+40 \%{ }^{\mathrm{b}}$ & Overall & $\begin{array}{l}-7.8 \mathrm{ng} \mathrm{m}^{-3} \%^{-1} \\
(-17,-1.1)\end{array}$ & $\begin{array}{l}-312 \text { to } 312 \\
(-680 \text { to } 680)\end{array}$ \\
\hline Mixing Height & -20 to $+20 \%$ & Overall & $\begin{array}{l}-3.5 \mathrm{ng} \mathrm{m}^{-3} \%^{-1} \\
(-9.5,1)\end{array}$ & $\begin{array}{l}-70 \text { to } 70 \\
(-190 \text { to } 190)\end{array}$ \\
\hline
\end{tabular}

${ }^{\text {a }}$ IPCC, 2007

${ }^{\mathrm{b}}$ Based on IPCC A2 scenario

${ }^{\mathrm{c}}$ Based on IPCC temperature projections and predicted base-case average RH during the three modeled periods 
Table S1. (continued)

\begin{tabular}{|c|c|c|c|c|}
\hline $\begin{array}{l}\text { Meteorological } \\
\text { Parameter }\end{array}$ & $\begin{array}{l}\text { Expected } \\
\text { Change of } \\
\text { Parameter }\end{array}$ & $\begin{array}{l}\text { Process } \\
\text { Affected }\end{array}$ & $\begin{array}{l}\text { Predicted Sensitivity } \\
\text { Mean }(10 \%, 90 \%)\end{array}$ & $\begin{array}{l}\text { Expected Concentration } \\
\text { Change }\left(\mathrm{ng} \mathrm{m}^{-3}\right) \\
\text { Mean }(10 \%, 90 \%)\end{array}$ \\
\hline \multicolumn{5}{|c|}{ Winter Period } \\
\hline \multirow{4}{*}{ Temperature } & \multirow{4}{*}{$+1-5.5 \mathrm{~K}^{\mathrm{a}}$} & $\begin{array}{l}\text { BVOC } \\
\text { emissions }\end{array}$ & $\begin{array}{l}10 \mathrm{ng} \mathrm{m}^{-3} \mathrm{~K}^{-1} \\
(-0.2,23)\end{array}$ & $\begin{array}{l}10 \text { to } 55 \\
(-1.1 \text { to } 127)\end{array}$ \\
\hline & & $\begin{array}{l}\text { Aerosol } \\
\text { partitioning }\end{array}$ & $\begin{array}{l}-25 \mathrm{ng} \mathrm{m}^{-3} \mathrm{~K}^{-1} \\
(-73,13)\end{array}$ & $\begin{array}{l}-25 \text { to }-138 \\
(-402 \text { to } 72)\end{array}$ \\
\hline & & Reaction rates & $\begin{array}{l}13.3 \mathrm{ng} \mathrm{m}^{-3} \mathrm{~K}^{-1} \\
(-1.1,25)\end{array}$ & $\begin{array}{l}13.3 \text { to } 73 \\
(-6 \text { to } 138)\end{array}$ \\
\hline & & Overall & $\begin{array}{l}-6.5 \mathrm{ng} \mathrm{m}^{-3} \mathrm{~K}^{-1} \\
(-90,65)\end{array}$ & $\begin{array}{l}-6.5 \text { to }-36 \\
(-495 \text { to } 358)\end{array}$ \\
\hline \multirow[t]{2}{*}{ Wind Speed } & \multirow[t]{2}{*}{-10 to $10 \%{ }^{b}$} & $\begin{array}{l}\text { Overall } \\
\text { (constant } \\
\text { emissions) }\end{array}$ & $\begin{array}{l}-36.4 \mathrm{ng} \mathrm{m}^{-3} \%^{-1} \\
(-83,-4.4)\end{array}$ & $\begin{array}{l}-364 \text { to } 364 \\
(-830 \text { to } 830)\end{array}$ \\
\hline & & $\begin{array}{l}\text { Sea salt } \\
\text { emissions }\end{array}$ & $\begin{array}{l}52.5 \mathrm{ng} \mathrm{m}^{-3} \\
(5,132)\end{array}$ & $\begin{array}{l}-525 \text { to } 525 \\
(-1320 \text { to } 1320)\end{array}$ \\
\hline $\begin{array}{l}\text { Absolute } \\
\text { humidity }\end{array}$ & +5 to $40 \%^{\mathrm{c}}$ & Overall & $\begin{array}{l}-7.6 \mathrm{ng} \mathrm{m}^{-3} \%^{-1} \\
(-35,9)\end{array}$ & $\begin{array}{l}-38 \text { to }-304 \\
(-1400 \text { to } 360)\end{array}$ \\
\hline $\begin{array}{l}\text { Precipitation } \\
\text { rate }\end{array}$ & -40 to $+40 \%{ }^{\mathrm{b}}$ & Overall & $\begin{array}{l}-12.2 \mathrm{ng} \mathrm{m}^{-3} \%^{-1} \\
(-33,-1.6)\end{array}$ & $\begin{array}{l}-488 \text { to } 488 \\
(-1320 \text { to } 1320)\end{array}$ \\
\hline $\begin{array}{l}\text { Precipitation } \\
\text { area }\end{array}$ & -40 to $+40 \%{ }^{b}$ & Overall & $\begin{array}{l}-7.3 \mathrm{ng} \mathrm{m}^{-3} \%^{-1} \\
(-15,-1.5)\end{array}$ & $\begin{array}{l}-292 \text { to } 292 \\
(-600 \text { to } 600)\end{array}$ \\
\hline Mixing Height & -20 to $+20 \%$ & Overall & $\begin{array}{l}-1.8 \mathrm{ng} \mathrm{m}^{-3} \%^{-1} \\
(-5,0.3)\end{array}$ & $\begin{array}{l}-36 \text { to } 36 \\
(-100 \text { to } 100)\end{array}$ \\
\hline
\end{tabular}

${ }^{\mathrm{a}}$ IPCC, 2007

${ }^{\mathrm{b}}$ Based on IPCC A2 scenario

${ }^{\mathrm{c}}$ Based on IPCC temperature projections and predicted base-case average RH during the three modeled periods. 
Table S1. (continued)

\begin{tabular}{|c|c|c|c|c|}
\hline $\begin{array}{l}\text { Meteorological } \\
\text { Parameter }\end{array}$ & $\begin{array}{l}\text { Expected } \\
\text { Change of } \\
\text { Parameter }\end{array}$ & $\begin{array}{l}\text { Process } \\
\text { Affected }\end{array}$ & $\begin{array}{l}\text { Predicted Sensitivity } \\
\text { Mean }(10 \%, 90 \%)\end{array}$ & $\begin{array}{l}\text { Expected Concentration } \\
\text { Change }\left(\mathrm{ng} \mathrm{m}^{-3}\right) \\
\text { Mean }(10 \%, 90 \%)\end{array}$ \\
\hline \multicolumn{5}{|c|}{ Fall Period } \\
\hline \multirow{4}{*}{ Temperature } & \multirow{4}{*}{$+1-5.5 \mathrm{~K}^{\mathrm{a}}$} & $\begin{array}{l}\text { BVOC } \\
\text { emissions }\end{array}$ & $\begin{array}{l}20.3 \mathrm{ng} \mathrm{m}^{-3} \mathrm{~K}^{-1} \\
(-1.4,52)\end{array}$ & $\begin{array}{l}20.3 \text { to } 112 \\
(-7.7 \text { to } 286)\end{array}$ \\
\hline & & $\begin{array}{l}\text { Aerosol } \\
\text { partitioning }\end{array}$ & $\begin{array}{l}-88 \mathrm{ng} \mathrm{m}^{-3} \mathrm{~K}^{-1} \\
(-285,2.3)\end{array}$ & $\begin{array}{l}-88 \text { to }-484 \\
(-1570 \text { to } 13)\end{array}$ \\
\hline & & Reaction rates & $\begin{array}{l}47.1 \mathrm{ng} \mathrm{m}^{-3} \mathrm{~K}^{-1} \\
(-0.1,115)\end{array}$ & $\begin{array}{l}47.1 \text { to } 259 \\
(-0.6 \text { to } 633)\end{array}$ \\
\hline & & Overall & $\begin{array}{l}-33 \mathrm{ng} \mathrm{m}^{-3} \mathrm{~K}^{-1} \\
(-210,85)\end{array}$ & $\begin{array}{l}-33 \text { to }-182 \\
(-1156 \text { to } 468)\end{array}$ \\
\hline \multirow[t]{2}{*}{ Wind Speed } & \multirow[t]{2}{*}{-10 to $10 \%{ }^{b}$} & $\begin{array}{l}\text { Overall } \\
\text { (constant } \\
\text { emissions) }\end{array}$ & $\begin{array}{l}-38.3 \mathrm{ng} \mathrm{m}^{-3} \%^{-1} \\
(-115,2)\end{array}$ & $\begin{array}{l}-383 \text { to } 383 \\
(-1150 \text { to } 1150)\end{array}$ \\
\hline & & $\begin{array}{l}\text { Sea salt } \\
\text { emissions }\end{array}$ & $\begin{array}{l}40 \mathrm{ng} \mathrm{m}^{-3} \\
(-1.9,123)\end{array}$ & $\begin{array}{l}-400 \text { to } 400 \\
(-1230 \text { to } 1230)\end{array}$ \\
\hline $\begin{array}{l}\text { Absolute } \\
\text { humidity }\end{array}$ & +5 to $40 \%^{\mathrm{c}}$ & Overall & $\begin{array}{l}11.5 \mathrm{ng} \mathrm{m}^{-3} \%^{-1} \\
(-21,57)\end{array}$ & $\begin{array}{l}58 \text { to } 460 \\
(-840 \text { to } 2280)\end{array}$ \\
\hline $\begin{array}{l}\text { Precipitation } \\
\text { rate }\end{array}$ & -40 to $+40 \%{ }^{\mathrm{b}}$ & Overall & $\begin{array}{l}-17.7 \mathrm{ng} \mathrm{m}^{-3} \%^{-1} \\
(-43,-3)\end{array}$ & $\begin{array}{l}-708 \text { to } 708 \\
(-1720 \text { to } 1720)\end{array}$ \\
\hline $\begin{array}{l}\text { Precipitation } \\
\text { area }\end{array}$ & -40 to $+40 \%{ }^{b}$ & Overall & $\begin{array}{l}-13.4 \mathrm{ng} \mathrm{m}^{-3} \%^{-1} \\
(-33,-3.3)\end{array}$ & $\begin{array}{l}-536 \text { to } 536 \\
(-1320 \text { to } 1320)\end{array}$ \\
\hline Mixing Height & -20 to $+20 \%$ & Overall & $\begin{array}{l}-2.2 \mathrm{ng} \mathrm{m}^{-3} \%^{-1} \\
(-6.5,1.1)\end{array}$ & $\begin{array}{l}-44 \text { to } 44 \\
(-130 \text { to } 130)\end{array}$ \\
\hline
\end{tabular}

${ }^{a}$ IPCC, 2007

${ }^{\mathrm{b}}$ Based on IPCC A2 scenario

${ }^{\mathrm{c}}$ Based on IPCC temperature projections and predicted base-case average $\mathrm{RH}$ during the three modeled periods. 

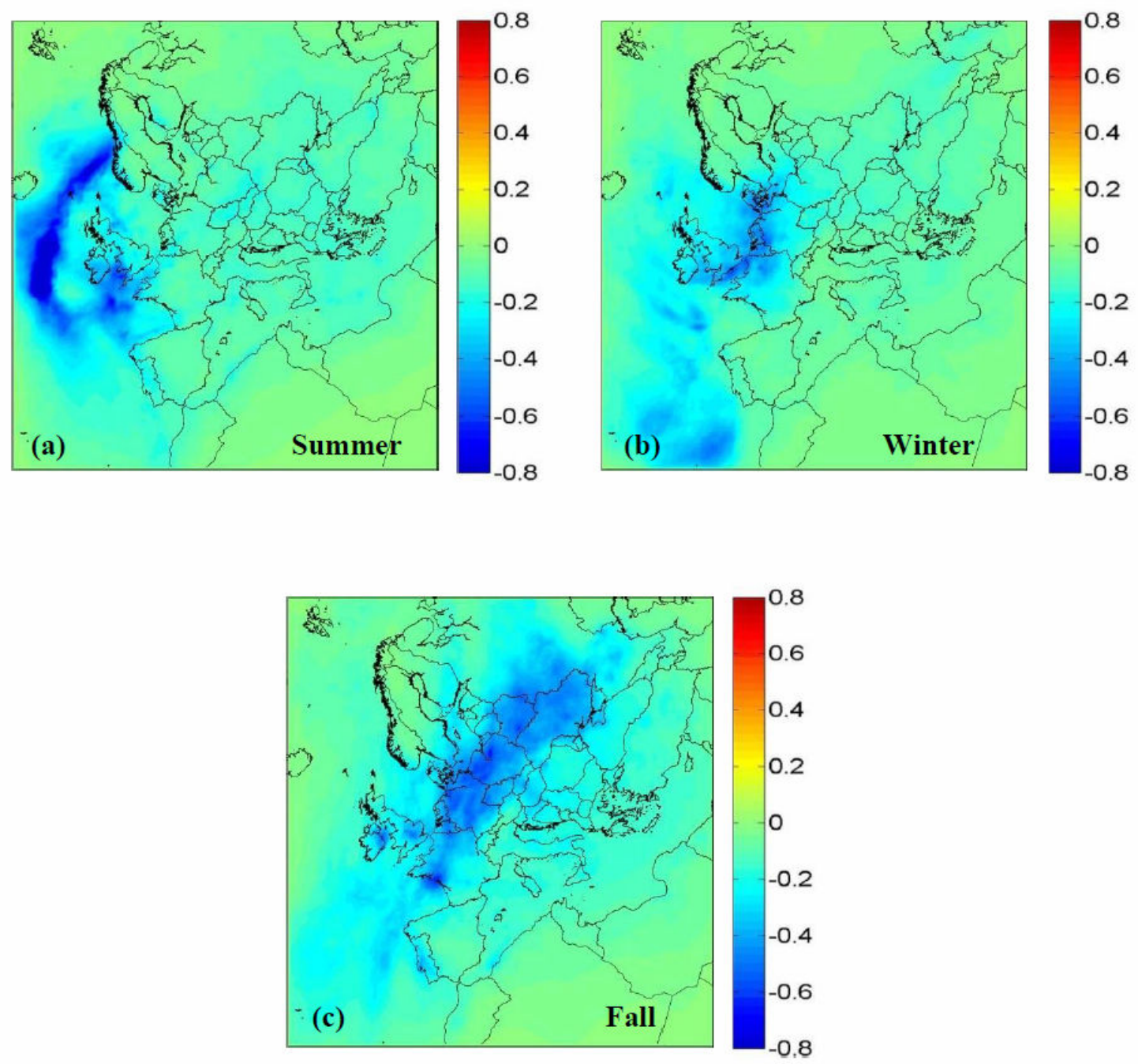

Figure S1. Predicted average change in ground-level concentrations $\left(\mu \mathrm{g} \mathrm{m}^{-3}\right)$ of total $\mathrm{PM}_{2.5}$ due to a $10 \%$ increase in precipitation rate during the modeled (a) summer, (b) winter and (c) fall periods. A positive value corresponds to an increase. 

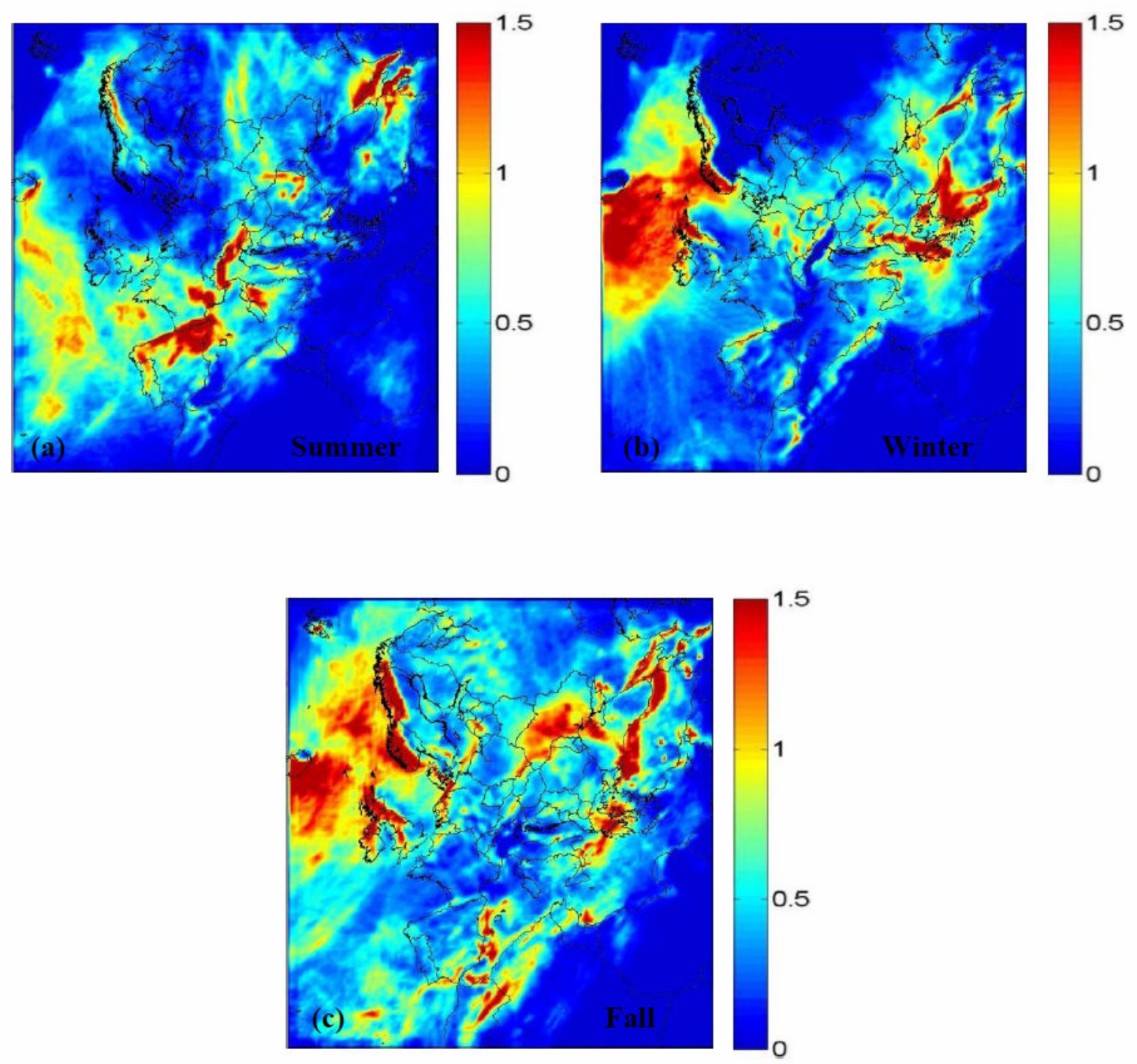

Figure S2. Predicted base case rainfall rate $\left(\mathrm{mm} \mathrm{month}^{-1}\right)$ during the modeled (a) summer, (b) winter and (c) fall periods. 

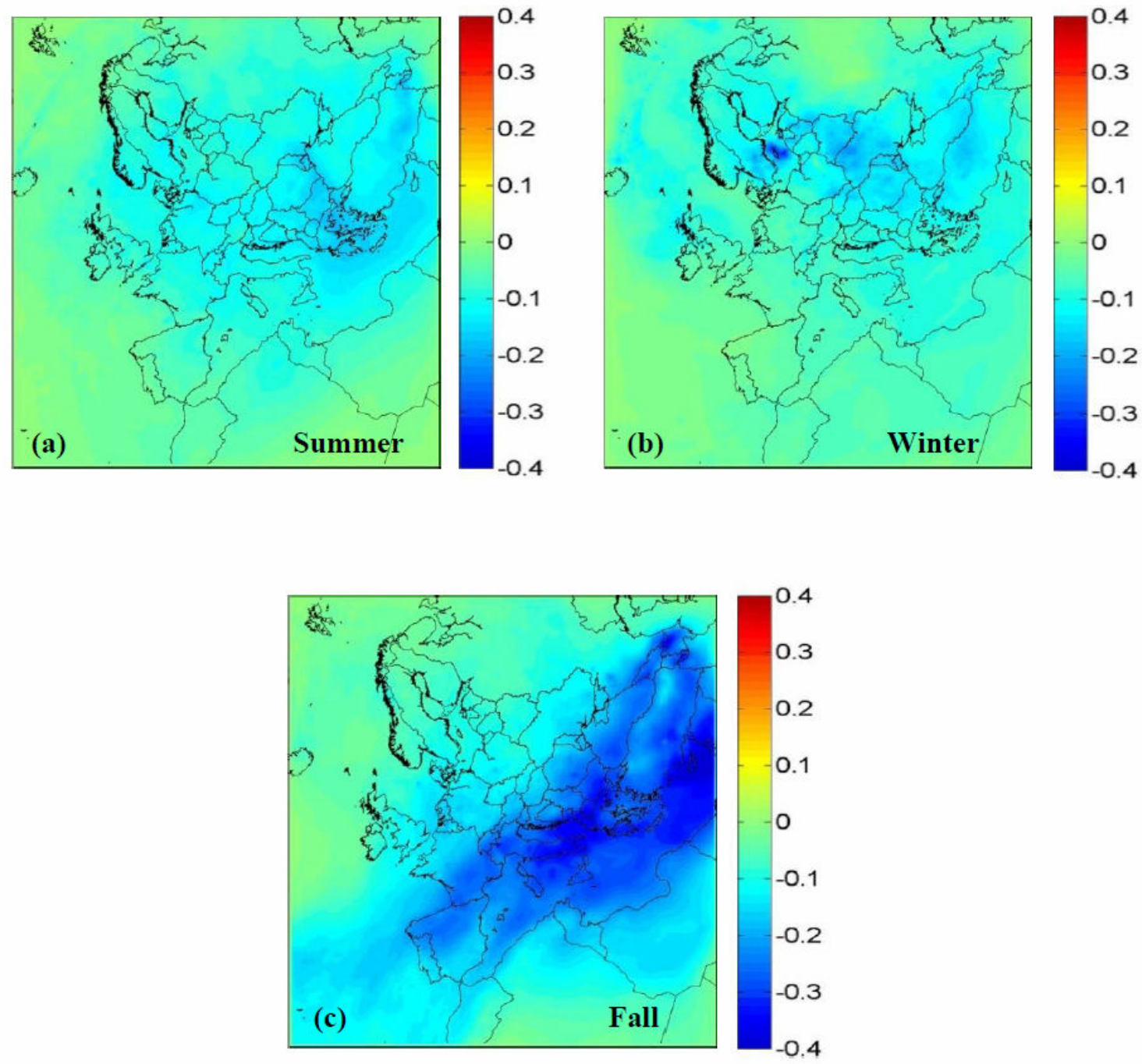

Figure S3. Predicted average change in ground-level concentrations $\left(\mu \mathrm{g} \mathrm{m}^{-3}\right)$ of total $\mathrm{PM}_{2.5}$ due to a $10 \%$ increase in area undergoing precipitation during the modeled (a) summer, (b) winter and (c) fall periods. A positive value corresponds to an increase. 

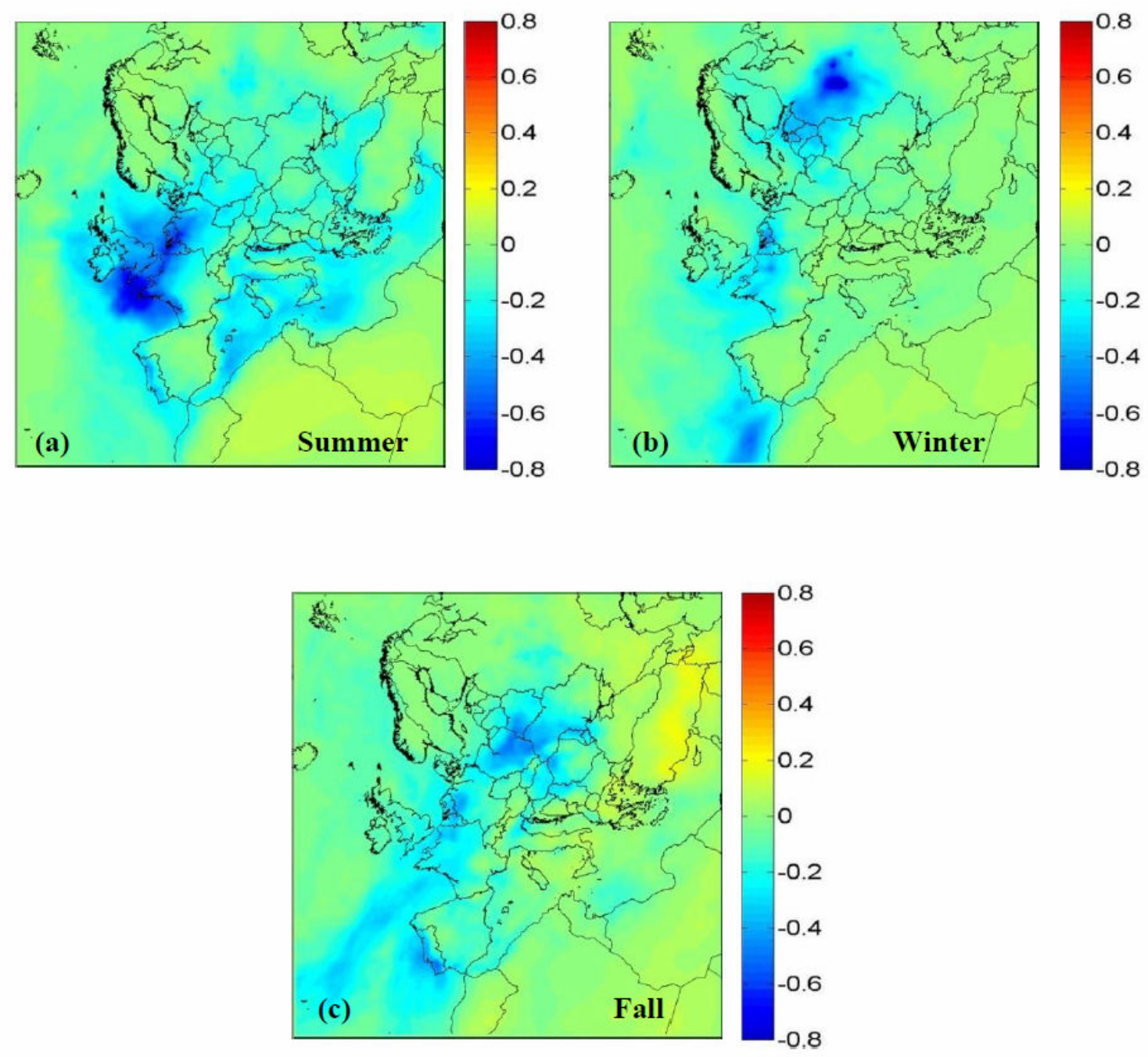

Figure S4. Predicted average change in ground-level concentrations $\left(\mu \mathrm{g} \mathrm{m}^{-3}\right)$ of total $\mathrm{PM}_{2.5}$ due to an increase of mixing height by one model layer (approximately $150 \mathrm{~m}$ ) during the modeled (a) summer, (b) winter and (c) fall periods. A positive value corresponds to an increase. 